\title{
Correlative Tomography for Additive Manufacturing of Biomedical Implants
}

\author{
B. Winiarski ${ }^{1,2}$, G. Pyka ${ }^{1}$, M. Benedatti ${ }^{3}$, T.L. Burnett ${ }^{2}$, D. Laeveren ${ }^{4}$, M. Dallago ${ }^{3}$ and P.J. Withers ${ }^{2}$ \\ 1. Thermo Fisher Scientific (FEI Czech Republic s.r.o.), Vlastimila Pecha 12, Brno, Czech Republic \\ 2. Henry Moseley X-ray Imaging Facility, School of Materials, University of Manchester, U.K. \\ 3. Department of Industrial Engineering, University of Trento, Via Sommarive 9, Trento, Italy \\ 4. Thermo Fisher Scientific - FEI, Achtseweg Noord 5, Bldg 5651 GG, Eindhoven, The Netherlands
}

Correlative tomography [1] is a concept/workflow of spatial registration in two and three dimensions (2D and 3D) of many imaging modalities - light microscopy (LM), electron/ion microscopy (EM, IM), X-Ray tomography, 2D/3D EBSD [2], EDS, Raman, etc.) - that allows various types of information, and at different lenghscale, to be collected for the same region of interest (ROI). In soft tissue biology, a 2D correlation of EM and LM s an invaluable tool to study the interactions of viruses with cells, and the ultrastructural changes induced in host cells by virus infection [3]. While biological materials are generally characterized as having complex 3D hierarchical microstructures [4] giving rise to interesting combinations of anisotropic mechanical properties that, in many cases, surpass those of manmade materials. A better understanding of these hierarchical structures and engineering of biomedical materials and structures requires a multiscale correlative imaging approach, which brings together 3D multimodal information at each length scale [1] often aided with temporal (4D) imaging [5].

Biocompatible titanium and its alloys are widely used in orthopaedic surgery for the replacement and stabilization of damaged bone tissue because of the high specific strength, low stiffness and high corrosion and fatigue resistance. Porous metal implants/scaffolds, additively manufactured (AM) by electron beam melting or selective laser melting (SLM), having an interconnected pore structure are of particular interest due to their potential ability to facilitate tissue ingrowth deep within the porous network and therefore present the possibility for reducing the stiffness mismatch between the loadbearing metal implant and bone [6]. Biomedical engineering uses computer-aided design models (CAD) and finite-element analysis (FEA) together with AM methods to design and reproduce scaffolds with controlled topology, porosity, pore shape and size, interconnectivity and mechanical properties. Nevertheless, AM processing conditions e.g. laser power, laser scanning speed, etc., and material impurities may have a significant impact on the scaffold morphology and mechanical performance leading to large deviations from the designed parameters [7]. Thus in order to precisely tailor the morphology and mechanical performance of implants, the information about actual geometry and microstructural defects needs to be feed back to the CAD/FEA model.

In this contribution we sketch the designing-manufacturing-testing feedback loop that uses correlative multiscale tomography workflow as an essential component for Biomedical Engineering Design. In this interactive design process by understanding the surface roughness we can optimize the biocompatibility and by helping optimize ALM process we can improve the mechanical properties. As a practical example, we study at different length scale and imaging modalities, morphology and microstructural features and imperfections of Ti-6Al-4V cellular orthopaedic scaffold produced by SLM from a CAD model. We use FEI Heliscan micro X-Ray Computed Tomography system and Helios Plasma FIB SEM microscope to facilitate the correlative multiscale tomography workflow and close the designingmanufacturing-testing feedback loop (Figure 1A). The loop consists three major blocks: I) design and numerical modeling (for simple structures this can be abandoned as indicated by procedure step ' $\mathrm{g}$ ' in Fig. 1A) ; II) AM manufacturing and mechanical testing (for not load-bearing structures this can be 
abandoned as indicated by procedure step ' $h$ ' in Fig. 1A); III) correlative multiscale tomography workflow. Here, a Plasma FIB-SEM not only can facilitate number of 2D, 3D imaging modalities (EBSD, EDS, SEM see inset in Fig. 1E) and residual stress mapping with micro-hole drilling (see inset in Fig. 1E), but also is able to excise sub-millimeter size samples (Fig. 1E and 1F) for further study and ROI correlation at smaller scale using micro XCT (as indicated by procedure step 'i' in Fig. 1A), PFIBSEM 3D imaging modalities, TEM or other techniques. The workflow and feedback loop is flexible and allows for: (a) smart information handling, e.g. feedback can be passed to different procedure steps (feedback loops ' 3 ' and '7' from XCT, and '4' and '8' from PFIB-SEM); (b) perform various procedure steps, e.g. temporal study (the procedure step ' $d$ ' and feedback loop ' 11 ') with the feedback to CAD/FEA procedure step (loop ' 5 '); (c) redesign material microstructure and surface topography (procedure 'h' and/or 'c,d' and loop '7').

We demonstrated the feasibility of FEI Heliscan micro X-Ray CT system and Helios Plasma FIB - SEM microscope combo as an ideal research instruments for correlative multiscale tomography analyses of biomedical materials and structures. PFIB system allowed for site-specific preparation and lift-out of massive sub-milometer blocks of material required for further 3D high resolution study. Presented designing-manufacturing-testing feedback loop has a potential for application in other bottom-up manufacturing methods.

References:

[1] Burnett TL et al, Scientific Reports 4 (2014), p. 4711.

[2] Burnett TL et al, Ultramicroscopy 161 (2016), p. 119.

[3] Bykov YS et al, FEBS Letters 590(13) (2016), p. 1877.

[4] Fratzl P, Weinkamer R. Prog. Mater. Sci. 52 (2007), p. 1263.

[5] Bradley RS, and Withers PJ. MRS Bulletin 41 (2016), p. 549.

[6] Ryan G et al, Biomaterials 27 (2006), p. 2651.

[7] Taniguchi S et al, Acta Biomaterialia 30 (2016) p. 345.

(A)

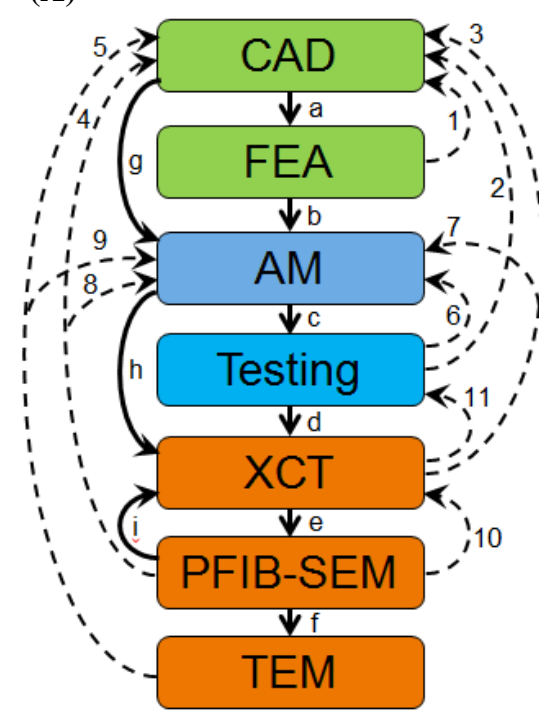

(B)
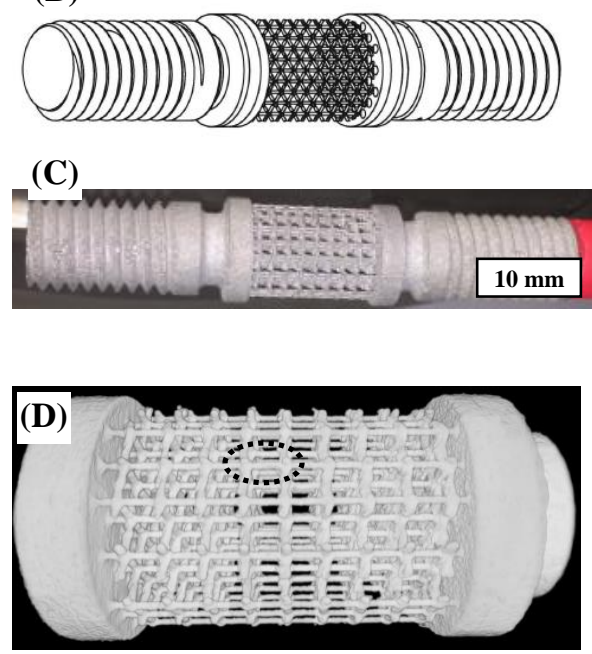
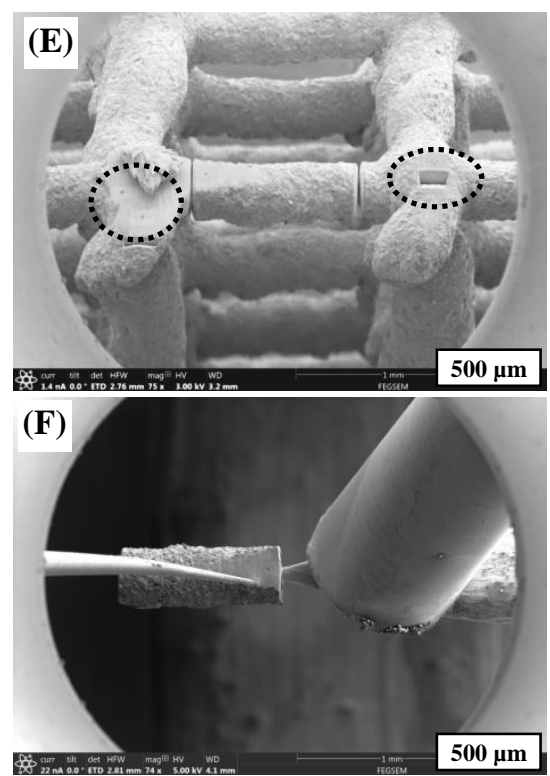

Figure 1. A) Shows correlative multiscale tomography for Biomedical Engineering Design, where indicate procedure steps (a-i) and dashed lines draws possible flow of feedback information (1-11). B) CAD model of the scaffold; C) SLM manufactured scaffold; D) 3D reconstructed central section from XCT data with marked region for further study at smaller length scale; E) ligament prepared for lift-out with EasyLift; dashed ovals mark regions where residual stresses were mapped with micro-hole drilling (left), and where cross-section was milled (right); F) ligament positioned on a pin for further high resolution XCT study. 